\title{
A Real-World Observational Study of Patients with Advanced Melanoma Receiving First-Line Ipilimumab in a Community Practice Setting
}

\author{
Debra A. Patt' ${ }^{1}$ Debra Rembert ${ }^{1}$, Menaka Bhor ${ }^{1}$, Debajyoti Bhowmik' ${ }^{1}$, Sumati A. Rao ${ }^{*}$ \\ ${ }^{1}$ Health Economics and Outcomes Research, McKesson Specialty Health, The Woodlands, TX, USA \\ ${ }^{2}$ Health Economics and Outcomes Research, Bristol-Myers Squibb, Plainsboro, NJ, USA \\ Email: sumati.rao@bms.com
}

Received 13 August 2014; revised 10 September 2014; accepted 5 October 2014

Copyright (C) 2014 by authors and Scientific Research Publishing Inc.

This work is licensed under the Creative Commons Attribution International License (CC BY). http://creativecommons.org/licenses/by/4.0/

\section{(c) (i) Open Access}

\section{Abstract}

Background: Following approval of ipilimumab, this observational cohort study (CA184-332) was initiated to describe patient and disease characteristics, patterns of care, survival, and adverse events (AEs) in advanced melanoma (AM) patients treated with first-line ipilimumab in realworld US community practice. Methods: Adult patients with treatment-naïve AM who received $\geq 1$ dose of ipilimumab $3 \mathrm{mg} / \mathrm{kg}$ between April 2011 and September 2012 were retrospectively identified at US Oncology sites. Clinical data were abstracted from patient medical records. Results: Median age of the 157 patient cohorts was 66 years (range 21 - 91). 68.2\% were male, and 90.5\% had a cutaneous primary site. At ipilimumab initiation, $80.9 \%$ of patients had an ECOG performance status of 0 or $1 ; 54.1 \%$ were stage M1c; $34.4 \%$ had brain metastases; $24.8 \%$ had elevated lactate dehydrogenase, and $13.4 \%$ were positive for BRAF mutation. All 4 cycles of ipilimumab were completed by $55.8 \%$ of patients. At a median follow-up of 8.5 months (range 2.9 - 15.0), median overall survival was 11.5 months (95\% CI: 8.9 - 16.6) and 1-year survival was $46.7 \%$ (95\% CI: 38.1 - 54.9). During ipilimumab treatment, AEs were experienced by $63.7 \%$ of patients. The most frequent AEs were gastrointestinal (41.4\%; diarrhea in $19.1 \%)$ and skin-related $(28.0 \%$; rash in $17.8 \%) ; 17.8 \%$ of patients had an AE that led to ipilimumab discontinuation. Conclusions: These real-world results are consistent with those from clinical trials and provide evidence supporting the effectiveness and safety of first-line ipilimumab $3 \mathrm{mg} / \mathrm{kg}$ monotherapy in patients with AM treated in a community practice setting.

${ }^{*}$ Corresponding author.

How to cite this paper: Patt, D.A., Rembert, D., Bhor, M., Bhowmik, D. and Rao, S.A. (2014) A Real-World Observational Study of Patients with Advanced Melanoma Receiving First-Line Ipilimumab in a Community Practice Setting. Journal of Cancer Therapy, 5, 1049-1058. http://dx.doi.org/10.4236/jct.2014.512110 


\section{Keywords}

\section{Ipilimumab, Immunotherapy, Community Practice, Overall Survival, Advanced Melanoma}

\section{Introduction}

The American Cancer Society estimates that 76,100 patients will be diagnosed with melanoma in 2014, making it the fifth most frequently diagnosed malignancy in the United States [1]. Moreover, the incidence rates of melanoma have been increasing in the United States for the past 40 years [2]. Although patients with melanoma overall have a 5-year relative survival rate of $91 \%$ [1], for patients diagnosed with or progressing to unresectable or metastatic (advanced) melanoma (AM), recent historical benchmark data estimated a median overall survival (OS) of 6.2 months (95\% CI: 5.9 - 6.5) and 1-year survival of 25.5\% (95\% CI: 23.6 - 27.4) [3]. Another recent systematic review estimated median OS for stage IV melanoma of $\sim 8$ months, with only $\sim 10 \%$ of patients surviving $>5$ years [4].

This dismal prognosis began to improve in 2010, with phase 3 trials of novel agents reporting, for the first time, statistically improved OS in patients with AM [5]-[7]. Several of these agents have now become available for use in routine clinical practice, with the first being the first-in-class immune checkpoint inhibitor ipilimumab $\left(\right.$ Yervoy $\left.^{\circledR}\right)$. A human monoclonal antibody, ipilimumab targets the cytotoxic T-lymphocyte-associated antigen 4 (CTLA-4) receptor, blocking its inhibitory mechanisms and thereby augmenting the natural T-lymphocyte-mediated antitumor immune response. Based on significant improvements in OS shown in two phase 3 randomized, double-blind trials in patients with AM, ipilimumab $3 \mathrm{mg} / \mathrm{kg}$ monotherapy every 3 weeks for 4 doses was approved in 2011 by the US Food and Drug Administration and the European Medicines Agency.

The initial ipilimumab pivotal trial was conducted in previously treated patients; ipilimumab $3 \mathrm{mg} / \mathrm{kg}$ prolonged OS, whether administered as monotherapy (median 10.1 months) or combined with a glycoprotein 100 (gp100) peptide vaccine (median 10.0 months), over gp100 alone (median 6.4 months; hazard ratio [HR] for death for ipilimumab alone vs gp100 alone, 0.66; $P=0.003$ ) [5]. Furthermore, there were $20.3 \%$ (45.6 vs 25.3 ) and $9.8 \%$ (23.5 vs 13.7 ) more patients alive in the ipilimumab-only arm than the gp100 arm at 1 year and 2 years, respectively. In the second phase 3 trial, patients with treatment-naïve disease receiving a concurrent regimen of ipilimumab $10 \mathrm{mg} / \mathrm{kg}$ and dacarbazine had improved OS over single-agent dacarbazine (median 11.2 vs 9.1 months; HR: 0.72; $P<0.001$ [ [6]. Higher survival rates were also seen in this trial in the patients treated with ipilimumab: $47.3 \%$ vs $36.3 \%$ at 1 year, $28.5 \%$ vs $17.9 \%$ at 2 years, and $20.8 \%$ vs $12.2 \%$ at 3 years. In both trials, ipilimumab was well tolerated and associated with a manageable safety profile. Adverse events (AEs) were primarily immune-related in nature, consistent with the mechanism of action of ipilimumab. Observations from these and other ipilimumab clinical trials resulted in product-specific AE management guidelines to mitigate the impact of immune-related AEs (irAEs) [8].

When new drugs are approved, data from use in real-world clinical settings may have considerable utility in confirming observations obtained within the controlled setting of phase 3 clinical trials. Shortly after ipilimumab was approved in the United States, we initiated this study (CA184-332), a multisite observational chart review, to gather such data, specifically in patients with AM treated with first-line ipilimumab in a real-world practice setting. The primary objectives were to describe 1) patient demographic and disease characteristics; 2) patterns of care; 3) survival outcomes; and 4) AEs of patients with treatment-naïve AM who were treated with ipilimumab $3 \mathrm{mg} / \mathrm{kg}$ monotherapy in a community practice setting. Here we report results for a cohort of patients for whom at least 12 months had elapsed since ipilimumab initiation at the time of this analysis; the study protocol calls for yearly updates of this cohort out to 4 years.

\section{Methods}

\subsection{Study Design}

CA184-332 was a retrospective electronic health record (EHR) observational cohort study of patients treated with first-line ipilimumab monotherapy in the clinical practices of the McKesson Specialty Health/US Oncology Network (MSH/USON). Patients in the study cohort were identified retrospectively from MSH/USON's 
iKnowMed (iKM) EHR system as having initiated first-line ipilimumab treatment for AM. Data were abstracted via programmatic query of the iKM system and EHR chart review, supplemented with data from the Social Security Death Master File. The study was submitted and approved through the US Oncology Institutional Review Board (IRB) process. Because this was a retrospective/observational study with IRB exempt/waiver status, patient consent was not required.

\subsection{Patients}

Eligible patients were required to have AM, be $\geq 18$ years old, have started ipilimumab monotherapy at $3 \mathrm{mg} / \mathrm{kg}$ in the first-line setting during April 1, 2011 to September 30, 2012 and received care at an MSH/USON Comprehensive Strategic Alliance Network site that used full iKM EHR capabilities over the entire study period. Exclusion criteria included prior systemic treatment for AM, current or pending enrollment in a clinical trial, and treatment for other cancers.

\subsection{Study Variables}

Variables to describe patient demographic and disease characteristics included gender, age, race, primary site at diagnosis, time since initial melanoma diagnosis, disease stage at start of ipilimumab treatment, location of metastases, ECOG performance status (PS), presence of brain metastases, serum lactate dehydrogenase (LDH) level, and BRAF mutation status. Variables to evaluate patterns of care included ipilimumab dosing, treatment delays and discontinuations, reasons for treatment termination, and concomitant and subsequent supportive and anticancer therapy. OS was the primary measure of treatment effectiveness and was defined as the time from start of ipilimumab until death from any cause. Safety was assessed by reported AEs. Because AE grade/intensity information, causality (e.g. treatment-related or not), and date of occurrence were not available in the iKM source data, AE data are reported irrespective of grade or causality. However, any AE reported that occurred during ipilimumab treatment was captured. Any AEs documented as serious AEs (SAEs) in patients' charts or leading to a hospital/emergency room visit or death were defined as SAEs.

\subsection{Statistical Analyses}

Demographics and baseline characteristics were assessed by descriptive statistics. Categorical variables (such as gender, M stage, ECOG PS, etc.) are reported as frequencies (\%). Continuous variables (such as age, time from initial melanoma diagnosis, etc.) are reported by median and range. Survival data were assessed by the Kaplan-Meier method and are reported as descriptive statistics (median OS and 1-year survival rate) with 95\% confidence intervals (95\% CI). An ad hoc, exploratory subgroup analysis of OS in patients with and without brain metastases was performed, but no further statistics were assessed on this comparison due to the exploratory nature of the analysis and the observational nature of the study.

\section{Results}

\subsection{Patient Demographics and Disease Characteristics}

A total of 157 patients were identified in the US Oncology iKM database with treatment-naïve AM who started ipilimumab therapy during the specified time period and otherwise met the eligibility criteria. The median age of the patient cohort was 66 years (range 21 - 91) years, and 68.2\% were male (Table 1). Of the 113 patients with ethnicity reported, $94.7 \%$ were white; $16.5 \%$ and $9.2 \%$ had comorbid diabetes and chronic pulmonary disease, respectively, and $6.6 \%$ had a history of myocardial infarction. The primary site at initial diagnosis was cutaneous in $90.5 \%$ of patients; of the remaining 15 patients, the primary site was ocular in 5 , mucosal in 3 , and unknown/missing in 7 . At the start of ipilimumab treatment, $44.0 \%$ and $36.9 \%$ of patients were ECOG 0 and 1 , respectively, $54.1 \%$ had stage IV M1c disease, 54 patients (34.4\%) had brain metastases, and 24.8\% had LDH greater than the upper limit of normal. Of the 118 patients for whom BRAF-mutation test results were known, $17.8 \%$ were positive. Median time from initial melanoma diagnosis to AM diagnosis was 8.0 months (range 0 352; 25 patients had a greater than 5-year gap between initial melanoma diagnosis and the start of ipilimumab treatment) and median time from AM diagnosis to start of ipilimumab treatment was 0.9 months (range 0 74.5). 
Table 1. Patient demographics and disease characteristics ${ }^{\mathrm{a}}$.

\begin{tabular}{|c|c|}
\hline Characteristic & Patients $(\mathrm{N}=157)$ \\
\hline Male, n (\%) & $107(68.2)$ \\
\hline Age, years, median (range) & $66(21-91)$ \\
\hline \multicolumn{2}{|l|}{ Race, $\mathbf{n}(\%)$} \\
\hline White & $107(68.2)$ \\
\hline Hispanic & $4(2.6)$ \\
\hline Black & $1(<1)$ \\
\hline Asian & $1(<1)$ \\
\hline Unknown/missing & $44(28.0)$ \\
\hline \multicolumn{2}{|l|}{ Primary site, n (\%) } \\
\hline Cutaneous & $142(90.5)$ \\
\hline Ocular & $5(3.2)$ \\
\hline Mucosal & $3(1.9)$ \\
\hline Unknown/missing & $7(4.5)$ \\
\hline \multicolumn{2}{|l|}{ Stage, $n(\%)^{c}$} \\
\hline M1a & $34(21.7)$ \\
\hline M1b & $35(22.3)$ \\
\hline M1c & $85(54.1)$ \\
\hline Unknown/missing & $3(1.9)$ \\
\hline \multicolumn{2}{|l|}{ ECOG performance status, n (\%) } \\
\hline 0 & $69(44.0)$ \\
\hline 1 & 58 (36.9) \\
\hline 2 & $13(8.3)$ \\
\hline 3 & $1(<1)$ \\
\hline Unknown/missing & $16(10.2)$ \\
\hline \multicolumn{2}{|l|}{ Metastases, n (\%) ${ }^{d}$} \\
\hline Lung & $80(51.0)$ \\
\hline In transit & $74(47.1)$ \\
\hline Liver & $65(41.4)$ \\
\hline Brain & $54(34.4)$ \\
\hline Subcutaneous tissues & 37 (23.6) \\
\hline Distant skin & $18(11.5)$ \\
\hline Adrenal & $17(10.8)$ \\
\hline Gastrointestinal & $14(8.9)$ \\
\hline Spleen & $4(2.6)$ \\
\hline \multicolumn{2}{|l|}{ LDH, n (\%) } \\
\hline Elevated & 39 (24.8) \\
\hline Normal & $86(54.8)$ \\
\hline Unknown/missing & $32(20.4)$ \\
\hline \multicolumn{2}{|l|}{ BRAF mutation status, $n(\%)^{e}$} \\
\hline Negative & 97 (61.8) \\
\hline Positive & $21(13.4)$ \\
\hline Unknown/missing & $39(24.8)$ \\
\hline
\end{tabular}

${ }^{a}$ At start of ipilimumab treatment unless otherwise noted. ${ }^{b}$ At initial diagnosis of melanoma. ${ }^{c}$ At diagnosis of advanced melanoma. ${ }^{\mathrm{d}}$ Patients could have multiple sites of

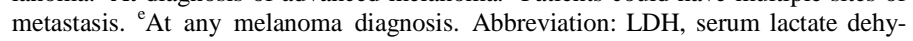
drogenase. 


\subsection{Patterns of Care}

All 4 cycles of first-line ipilimumab therapy were completed by $55.8 \%$ of patients (Table 2 ). The most frequent reasons given for ipilimumab discontinuation were toxicity $(17.7 \%)$, disease progression/loss of response (16.3\%), death (4.1\%), and patient choice (1.4\%). At least 1 dose of ipilimumab therapy was delayed in $38.0 \%$ of patients, and $22.5 \%$ had $>1$ dose delay. Supportive therapy received included steroids in $36.0 \%$ of patients, antidiarrheal agents in $16.3 \%$, and infliximab in 2 patients (1.3\%). In addition, $69.9 \%$ of patients received radiotherapy, and $43.8 \%$ received hospice care. One additional course (second-line therapy) of anticancer therapy subsequent to ipilimumab therapy was received by 40 patients (25.5\%), and two additional courses (second- and third-line therapy) by 9 patients (5.7\%).

\subsection{Survival}

At a median follow-up of 8.5 months (interquartile range: 2.9 - 15.0) from the start of ipilimumab treatment, 83 patients (52.2\%) had died. The median OS was 11.5 months (95\% CI: 8.9 - 16.6) and 1-year survival was 46.7\% (95\% CI: 38.1 - 54.9\%) (Figure 1(a)). Included in the 83 patients who died were 49 of 103 patients (47.6\%) without brain metastases and 34 of 54 patients (63.0\%) with brain metastases. The ad hoc exploratory survival analysis of patients without and with brain metastases estimated median OS for these subgroups of 14.1 (95\% CI: 9.7 - not reached) and 7.0 months (95\% CI: 5.1 - 12.8), respectively (Figure 1(b)).

\subsection{Safety}

During ipilimumab therapy, at least one AE was experienced by $63.7 \%$ of patients (Table 3 ). The most frequent AEs $(\geq 10 \%)$ were gastrointestinal (41.4\%), with diarrhea (19.1\%) the most frequent of these, and skin-related (28.0\%), of which rash $(17.8 \%)$ was most frequently reported. Neurological, endocrine, and liver AEs were reported in 14 (8.9\%), 6 (3.8\%), and 3 (1.9\%) patients, respectively. Of the 83 deaths that occurred by the end of follow-up, 78 were reported as related to melanoma (progression), 1 was reported as not related to melanoma, 1 patient died from acute myocardial infarction, and the cause of death in 3 patients was unknown. There was an SAE in 46 patients (29.5\%), and 28 patients (17.8\%) had an AE that led to discontinuation of ipilimumab treatment.

\section{Discussion}

This multisite, retrospective, observational chart review study provides real-world evidence supporting the

Table 2. First-line ipilimumab dosing and associated therapy.

\begin{tabular}{|c|c|}
\hline Type of therapy & Patients $(\mathrm{N}=157)$ \\
\hline \multicolumn{2}{|l|}{ First-line ipilimumab $^{a}$} \\
\hline Completed all 4 cycles of first-line therapy & $82(55.8)$ \\
\hline Number of doses per patient, median (range) ${ }^{b, c}$ & $4(1-8)$ \\
\hline Experienced $\geq 1$ dose interruption, $\mathrm{n}(\%)^{\mathrm{b}}$ & $54(38.0)$ \\
\hline Received at least 4 doses, $\mathrm{n}(\%)^{\mathrm{d}}$ & $92(64.8)$ \\
\hline \multicolumn{2}{|l|}{ Supportive therapy ${ }^{e, f}$} \\
\hline Steroids & $55(36.0)$ \\
\hline Antidiarrheal & $25(16.3)$ \\
\hline Infliximab & $2(1.3)$ \\
\hline Radiotherapy ${ }^{\text {e,f }}$ & 107 (69.9) \\
\hline Hospice care $e^{e, f}$ & $67(43.8)$ \\
\hline
\end{tabular}

${ }^{\mathrm{a} A p p r o v e d} 3 \mathrm{mg} / \mathrm{kg}$ monotherapy q3w $\times 4{ }^{\mathrm{b}}{ }^{\mathrm{b}}$ These dosing details known for $142 \mathrm{pa}-$ tients. ${ }^{\mathrm{C}}$ Two patients received 5 doses and 3 patients received 8 doses of ipilimumab. ${ }^{\mathrm{d}}$ Includes initial ipilimumab treatment and retreatment. ${ }^{\mathrm{e}}$ Information available for 153 patients. ${ }^{\mathrm{f}}$ Received at any time during the study period. 


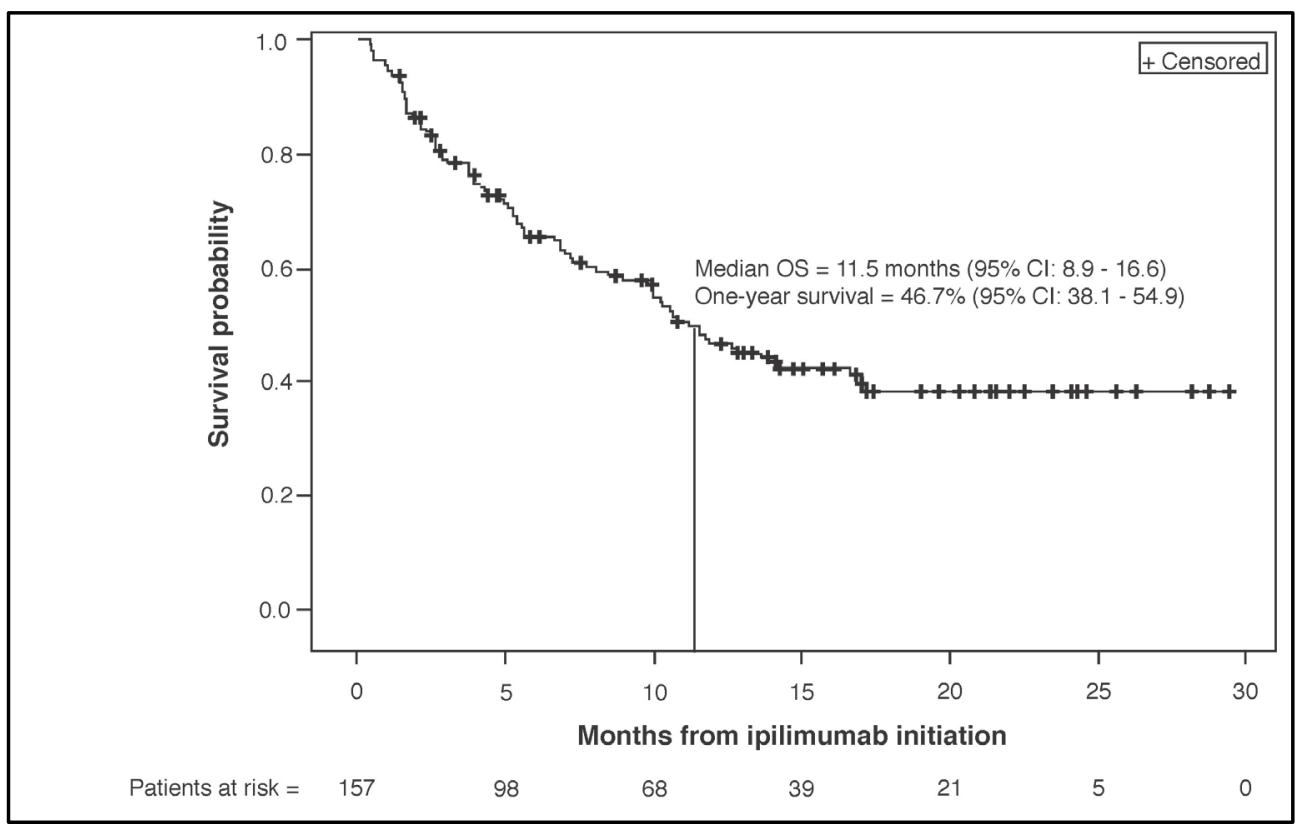

(a)

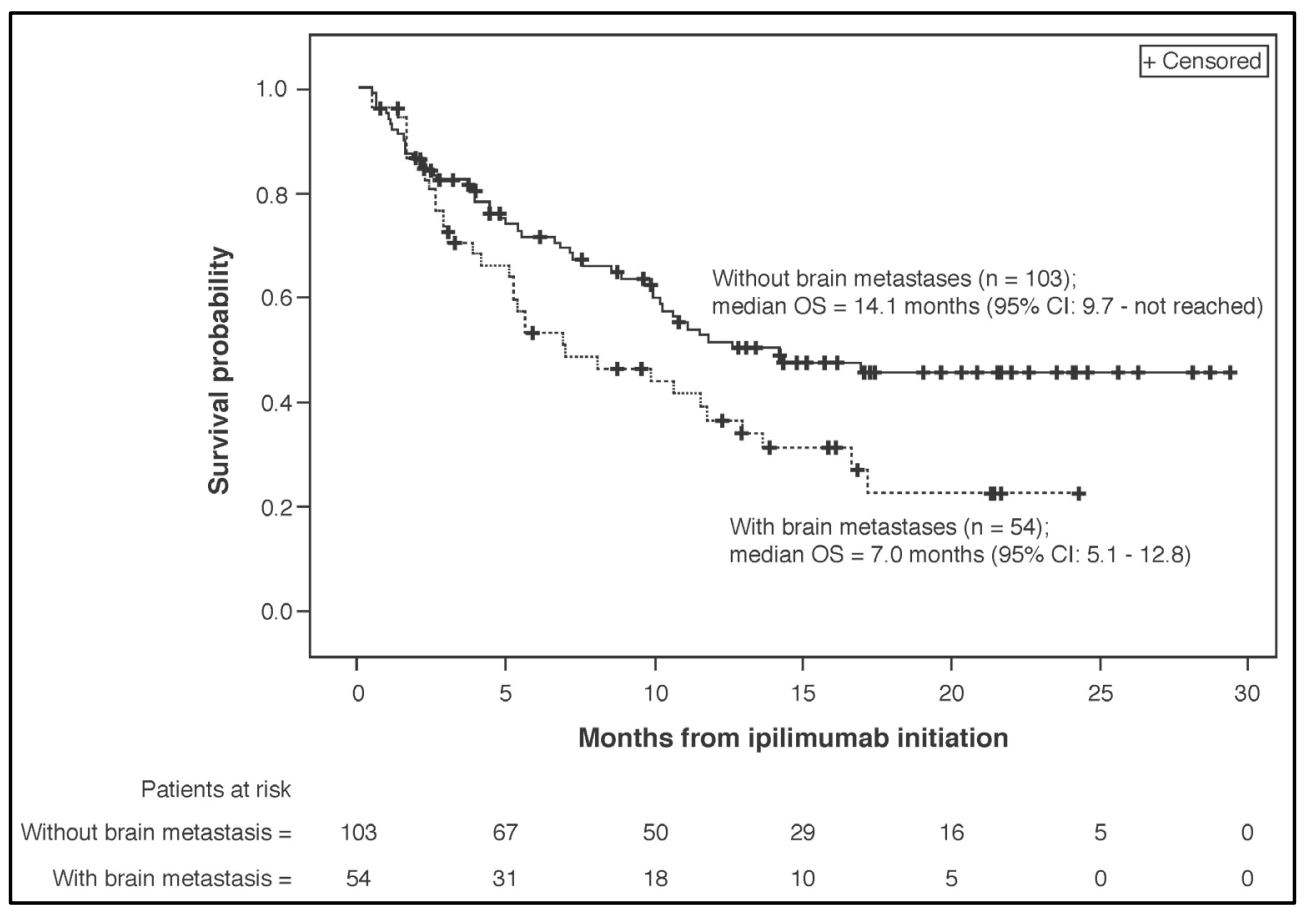

(b)

Figure 1. (a) Kaplan-Meier plot for overall survival from ipilimumab initiation ( $\mathrm{N}=157)$. (b) Ad hoc, exploratory Kaplan-Meier plot for overall survival from ipilimumab initiation for subgroups of patients without $(n=103)$ and with brain metastases $(n=54)$. Abbreviations: OS, overall survival; CI, confidence interval.

effectiveness and safety of the approved ipilimumab $3 \mathrm{mg} / \mathrm{kg}$ monotherapy every 3 weeks for 4 doses administered to patients with treatment-naïve AM in a US community oncology practice setting. Patients treated outside the controlled setting of a randomized clinical trial are expected to exhibit greater heterogeneity in demographic and disease characteristics than those in a clinical trial population, and this increased heterogeneity could result 
Table 3. Summary of adverse events reported in patients receiving first-line ipilimumab ${ }^{\mathrm{a}}$.

\begin{tabular}{|c|c|}
\hline Adverse event ${ }^{\mathrm{b}}$ & Patients, n (\%) (N = 157) \\
\hline All & $100(63.7)$ \\
\hline Gastrointestinal & $65(41.4)$ \\
\hline Diarrhea & 30 (19.1) \\
\hline Nausea & $9(5.7)$ \\
\hline Colitis & $8(5.1)$ \\
\hline Skin & $44(28.0)$ \\
\hline Rash & $28(17.8)$ \\
\hline Neurological & $14(8.9)$ \\
\hline Endocrine & $6(3.8)$ \\
\hline Liver & $3(1.9)$ \\
\hline Serious adverse events & $46(29.5)$ \\
\hline Adverse event leading to discontinuation & $28(17.8)$ \\
\hline
\end{tabular}

approved $3 \mathrm{mg} / \mathrm{kg}$ monotherapy q3w $\times 4$. ${ }^{\mathrm{b}}$ Information on intensity (grade) and causality not available from data source.

in poorer outcomes. This was not the case for this study, in which the observed survival (median OS of 11.5 months and 1-year survival of 46.7\%) is consistent with that reported in the ipilimumab pivotal phase 3 trials (median OS of 10.1 and 11.2 months and 1-year survival of $45.6 \%$ and $47.3 \%$ ) [5] [6]. Our results are also consistent with those of the two small phase 2 trials to report on ipilimumab monotherapy at the $3 \mathrm{mg} / \mathrm{kg}$ dose in treatment-naïve patients. In the first trial, 32 patients with chemotherapy-naïve AM had a median OS of 11.4 months and 1-year survival of 45\% [9]; in the second, 40 patients had a median OS of 12.9 months and 1-year survival of $60.9 \%$ [10].

The consistency of these survival results is notable given that $34.4 \%$ of patients in our observational cohort had brain metastases, a factor known to be related to poor prognosis. Only $12.1 \%$ of patients in the previously treated population in the phase 3 MDX010-20 trial had brain metastases [5], and none (brain metastases were excluded) were present in the treatment-naïve population in the phase 3 CA184-024 trial [6]. In addition, the populations treated in the two phase 3 trials were younger, with a mean age of 56.2 years across all arms in MDX010-20 [5] and 57.5 and 56.4 years in the two arms of CA184-024 [6], compared to a mean age of 64 years in our cohort. Importantly, the planned yearly survival updates of this cohort should provide real-world data on whether long-term survival is also consistent with the durable long-term survival ( $>3$ - 5 years) now shown for $20 \%$ - 26\% of patients in extended follow-up across the ipilimumab clinical trials [11].

Prior studies have demonstrated that brain metastases in patients with AM are, as mentioned above, highly prognostic for worse OS regardless of treatment received [3] [12], and more effective treatments for brain metastases in patients with AM is a well-recognized clinical need [13] [14]. Our ad hoc, exploratory OS analysis of the subgroups with and without brain metastases did show the expected poorer survival in patients with brain metastases (see Figure 1(b)). However, the median OS of 7.0 months for this subgroup still compares favorably with the historical benchmark median OS of 6.2 months estimated for patients with AM overall [3]. This exploratory result is also consistent with the median OS demonstrated in an open-label, phase 2 study of ipilimumab in patients with AM that had metastasized to the brain. In that study, which included 51 patients with asymptomatic brain metastases and 21 patients who were symptomatic but on a stable steroid dose, median OS after ipilimumab $10 \mathrm{mg} / \mathrm{kg}$ induction and maintenance monotherapy was 7 months and 4 months, respectively [13]. In another open-label, phase 2 trial, 10 of 20 AM patients with asymptomatic brain metastases achieved disease control and 54.2\% were alive at 1 year after receiving ipilimumab $10 \mathrm{mg} / \mathrm{kg}$ (4 doses) combined with fotemustine chemotherapy; at a median follow up of 10.8 months, median OS for these 20 patients was 13.4 months [14]. While this limited clinical experience shows that ipilimumab has activity in AM patients with brain metastases, particularly in those with asymptomatic brain metastases, prospective clinical trial data are needed to more clearly define its clinical activity in this poorly addressed subpopulation of AM patients. Regardless, the available 
evidence importantly also suggests that, as with most therapy for AM, the clinical benefit of ipilimumab treatment is likely to be greatest in patients who receive it early after a diagnosis of AM, certainly prior to progression to symptomatic brain metastases.

It should be noted that the frequency of BRAF mutations reported here $(17.8 \%$ of patients tested were BRAF-mutation positive) is lower than the $40 \%-60 \%$ expected in a population of patients with primarily cutaneous melanomas [7]. This finding may reflect physicians' preference to treat BRAF-mutated patients, particularly those with poor prognosis disease, with BRAF inhibitors. Whether patients with BRAF mutation-positive tumors should receive MAPK inhibitors or ipilimumab as first-line systemic therapy remains an area of uncertainty where prospective studies are ongoing to inform clinical practice [15]. Retrospective data suggest that while response to a BRAF inhibitor is similar pre- and post-ipilimumab, response to ipilimumab may be improved pre- versus post-BRAF inhibitor [16]. Based on this evidence, some authors recommend using ipilimumab before a BRAF inhibitor, particularly in an initial setting of asymptomatic or low-volume disease; in the future, however, a potential strategy may be to start with targeted therapy and then switch to immunotherapy at the time of best response (before failure occurs) [16] [17].

Supporting the generalizability of our results, the US community oncology practices affiliated with MSH/ USON cover a wide geographical area in the United States and the iKM EHR system is estimated to capture data on $10 \%$ of newly diagnosed cancer patients in the United States. Another multisite, retrospective, observational cohort study (CA184-338) was conducted with the same objectives during the same timeframe. In contrast to our study, however, the CA184-338 study included patients treated primarily at specialized centers (Cytokine Working Group [CWG] or CWG-affiliated) [18]. Thus, patients in this second cohort were managed by academic clinicians who had extensive clinical trial experience with ipilimumab therapy, selection of appropriate patients, and irAE management, and who were supported by similarly experienced and dedicated clinical and research teams. For the 273-patient cohort receiving first-line ipilimumab in this second study, median OS was 14.5 months (95\% CI: 12.9 - 18.7) and 1-year survival was 59.2\% (95\% CI: 53.0 - 64.8). As noted, our cohort had a high proportion of patients with brain metastases (34.4\% compared with $12.1 \%$ in CA184-338), as well as fewer patients who received all 4 cycles of the first-line therapy (53.8\% compared with 77.7\% in CA184-338). Both of these differences could have contributed to the lower survival observed in our study relative to study CA184-338. In any case, despite the differences, the results of these two observational studies are independently consistent with those from the ipilimumab clinical trials, strongly suggesting that improved survival can be achieved with ipilimumab in the real-world treatment settings of both community and academic practice.

While our AE data are limited, ipilimumab appeared to be well tolerated in this treatment-naïve cohort, with $55.8 \%$ of patients receiving all 4 cycles of therapy. In the two ipilimumab-containing arms of the MDX010-20 trial in previously treated patients, all 4 cycles were received by $60.0 \%$ and $64.2 \%$ of patients [5]. As in the ipilimumab clinical trials, the most frequently reported AEs in this study occurred in the skin and gastrointestinal systems, and they occurred at comparable frequencies for all categories. Likely reflecting the use of the irAE management guidelines [8], 55 patients (36.0\%) received steroids as supportive therapy, with 2 patients (1.3\%) additionally treated with infliximab, presumably for irAEs not adequately controlled by steroid therapy.

This observational study is limited by its retrospective nature and is, therefore, subject to potential selection bias, which warrants caution when interpreting the results or directly comparing them to the results of interventional studies. Additionally, the analyses are limited by loss to follow-up, as well as missing or over- or underreported data, particularly with regard to AEs. Due to the setting of care (community oncology practices), the patterns of care may have differed to some degree from treatment patterns in general and may not reflect (as suggested by the higher survival seen in the parallel CA184-338 observational study) outcomes for patients treated at more experienced sites that may have participated in the ipilimumab clinical trial or expanded access programs. For the OS analysis, the censoring of patients not known to have died by the time of the data lock reduces the reliability of the OS results with increasing time, with an impact that is expected to decline with longer follow-up.

\section{Conclusion}

In conclusion, this report provides a real-world assessment of the approved ipilimumab $3 \mathrm{mg} / \mathrm{kg}$ as a first-line therapy in patients with AM treated in a community oncology practice setting. It also provides additional information on outcomes for AM patients with brain metastases, for whom clinical trial data are limited. The 
observed median OS of 11.5 months and 1-year survival of $46.7 \%$ suggest that the improved survival associated with ipilimumab in clinical trials is also achievable in a real-world community practice setting. While subject to the limitations of data obtained from observational studies, these results support the effectiveness and safety of ipilimumab used at the indicated 3-mg/kg dose and schedule as first-line therapy for patients with AM.

\section{Funding}

This study was funded by Bristol-Myers Squibb.

\section{Acknowledgements}

The authors take full responsibility for the content of this publication and confirm that it reflects their viewpoint and medical expertise. The authors also wish to acknowledge Schiffon Wong, John Penrod, Rahul Dhanda, Marianne Messina, Clara Chen, and Mark Yap for their work on the included analyses. Professional medical writing and editing support was provided by Jennifer Wietzke at Stem Scientific and funded by Bristol-Myers Squibb.

\section{References}

[1] American Cancer Society (2014) American Cancer Society: Cancer Facts \& Figures 2014. American Cancer Society, Atlanta.

[2] Surveillance Epidemiology and End Results: Cancer Statistics. http://seer.cancer.gov/statfacts/html/all.html

[3] Korn, E.L., Liu, P.Y., Lee, S.J., Chapman, J.A., Niedzwiecki, D., Suman, V.J., et al. (2008) Meta-Analysis of Phase II Cooperative Group Trials in Metastatic Stage IV Melanoma to Determine Progression-Free and Overall Survival Benchmarks for Future Phase II Trials. Journal of Clinical Oncology, 26, 527-534. http://dx.doi.org/10.1200/JCO.2007.12.7837

[4] Garbe, C., Eigentler, T.K., Keilholz, U., Hauschild, A. and Kirkwood, J.M. (2011) Systematic Review of Medical Treatment in Melanoma: Current Status and Future Prospects. The Oncologist, 16, 5-24. http://dx.doi.org/10.1634/theoncologist.2010-0190

[5] Hodi, F.S., O’Day, S.J., McDermott, D.F., McDermott, D.F., Weber, R.W., Sosman, J.A., Haanen, J.B., et al. (2010) Improved Survival with Ipilimumab in Patients with Metastatic Melanoma. New England Journal of Medicine, 363, 711-723. http://dx.doi.org/10.1056/NEJMoa1003466

[6] Robert, C., Thomas, L., Bondarenko, I., O’Day, S., Weber, J., Garbe, C., et al. (2011) Ipilimumab plus Dacarbazine for Previously Untreated Metastatic Melanoma. New England Journal of Medicine, 364, 2517-2526. http://dx.doi.org/10.1056/NEJMoa1104621

[7] Chapman, P.B., Hauschild, A., Robert, C., Haanen, J.B., Ascierto, P., Larkin, J., et al. (2011) Improved Survival with Vemurafenib in Melanoma with BRAF V600E Mutation. New England Journal of Medicine, 364, 2507-2516. http://dx.doi.org/10.1056/NEJMoa1103782

[8] Weber, J.S., Kähler, K.C. and Hauschild, A. (2012) Management of Immune-Related Adverse Events and Kinetics of Response with Ipilimumab. Journal of Clinical Oncology, 30, 2691-2697. http://dx.doi.org/10.1200/JCO.2012.41.6750

[9] Hersh, E.M., O’Day, S.J., Powderly, J., Khan, K.D., Pavlick, A.C., Cranmer, L.D., et al. (2011) A Phase II Multicenter Study of Ipilimumab with or without Dacarbazine in Chemotherapy-Naïve Patients with Advanced Melanoma. Investigational New Drugs, 29, 489-498. http://dx.doi.org/10.1007/s10637-009-9376-8

[10] Hamid, O., Schmidt, H., Nissan, A., Ridolfi, L., Aamdal, S., Hansson, J., et al. (2011) A Prospective Phase II Trial Exploring the Association between Tumor Microenvironment Biomarkers and Clinical Activity of Ipilimumab in Advanced Melanoma. Journal of Translational Medicine, 9, 204. http://dx.doi.org/10.1186/1479-5876-9-204

[11] Schadendorf, D., Hodi, F.S., Robert, C., Weber, J.S., Margolin, K., Hamid, O., et al. (2013) Pooled Analysis of LongTerm Survival Data from Phase II and Phase III Trials of Ipilimumab in Metastatic or Locally Advanced, Unresectable Melanoma. European Cancer Congress 2013 (ECCO-ESMO-ESTRO), Amsterdam, 27 September-1 October 2013, Abstract LBA 24.

[12] Damsky, W.E., Rosenbaum, L.E. and Bosenberg, M. (2010) Decoding Melanoma Metastasis. Cancers, 3, $126-163$. http://dx.doi.org/10.3390/cancers3010126

[13] Margolin, K., Ernstoff, M.S., Hamid, O., Lawrence, D., McDermott, D., Puzanov, I., et al. (2012) Ipilimumab in Patients with Melanoma and Brain Metastases: An Open-Label, Phase 2 Trial. The Lancet Oncology, 13, 459-465. http://dx.doi.org/10.1016/S1470-2045(12)70090-6 
[14] Di Giacomo, A.M., Ascierto, P.A., Pilla, L., Santinami, M., Ferrucci, P.F., Giannarelli, D., et al. (2012) Ipilimumab and Fotemustine in Patients with Advanced Melanoma (NIBIT M1): An Open-Label, Single-Arm Phase 2 Trial. The Lancet Oncology, 13, 879-886. http://dx.doi.org/10.1016/S1470-2045(12)70324-8

[15] Jang, S. and Atkins, M.B. (2013) Which Drug, and When, for Patients with BRAF-Mutant Melanoma? The Lancet Oncology, 14, e60-e69. http://dx.doi.org/10.1016/S1470-2045(12)70539-9

[16] Ackerman, A., Klein, O., McDermott, D.F., Wang, W., Ibrahim, N., Lawrence, D.P., et al. (2014) Outcomes of Patients with Metastatic Melanoma Treated with Immunotherapy Prior to or after BRAF Inhibitors. Cancer, 120, 16951701. http://dx.doi.org/10.1002/cncr.28620

[17] Ascierto, P.A. and Margolin, K. (2014) Ipilimumab before BRAF Inhibitor Treatment May Be More Beneficial than Vice Versa for the Majority of Patients with Advanced Melanoma. Cancer, 120, 1617-1619. http://dx.doi.org/10.1002/cncr.28622

[18] Margolin, K.A., Wong, S.L., Penrod, J.R., Song, J., Chang, I.F., Johnson, D.B., et al. (2013) Effectiveness and Safety of First-Line Ipilimumab $3 \mathrm{mg} / \mathrm{kg}$ Therapy for Advanced Melanoma: Evidence from a US Multisite Retrospective Chart Review. European Cancer Congress 2013 (ECCO-ESMO-ESTRO), Amsterdam, 27 September-1 October 2013, Abstract 3742. 
Scientific Research Publishing (SCIRP) is one of the largest Open Access journal publishers. It is currently publishing more than 200 open access, online, peer-reviewed journals covering a wide range of academic disciplines. SCIRP serves the worldwide academic communities and contributes to the progress and application of science with its publication.

Other selected journals from SCIRP are listed as below. Submit your manuscript to us via either submit@scirp.org or Online Submission Portal.
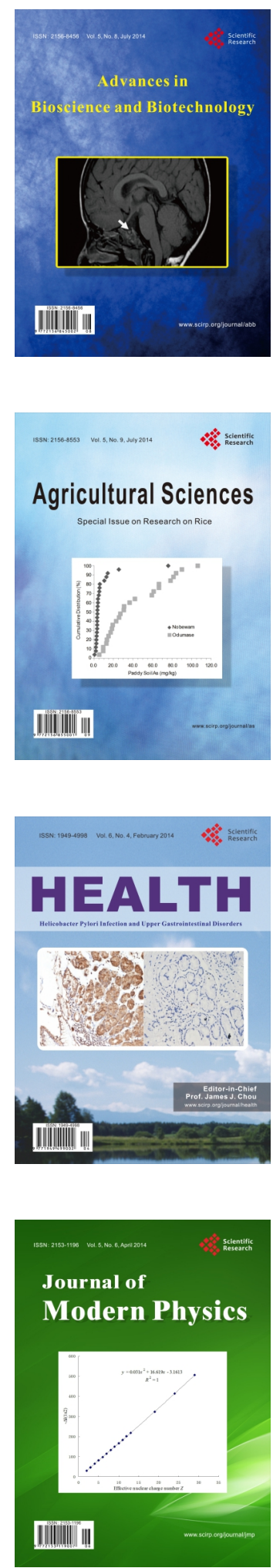
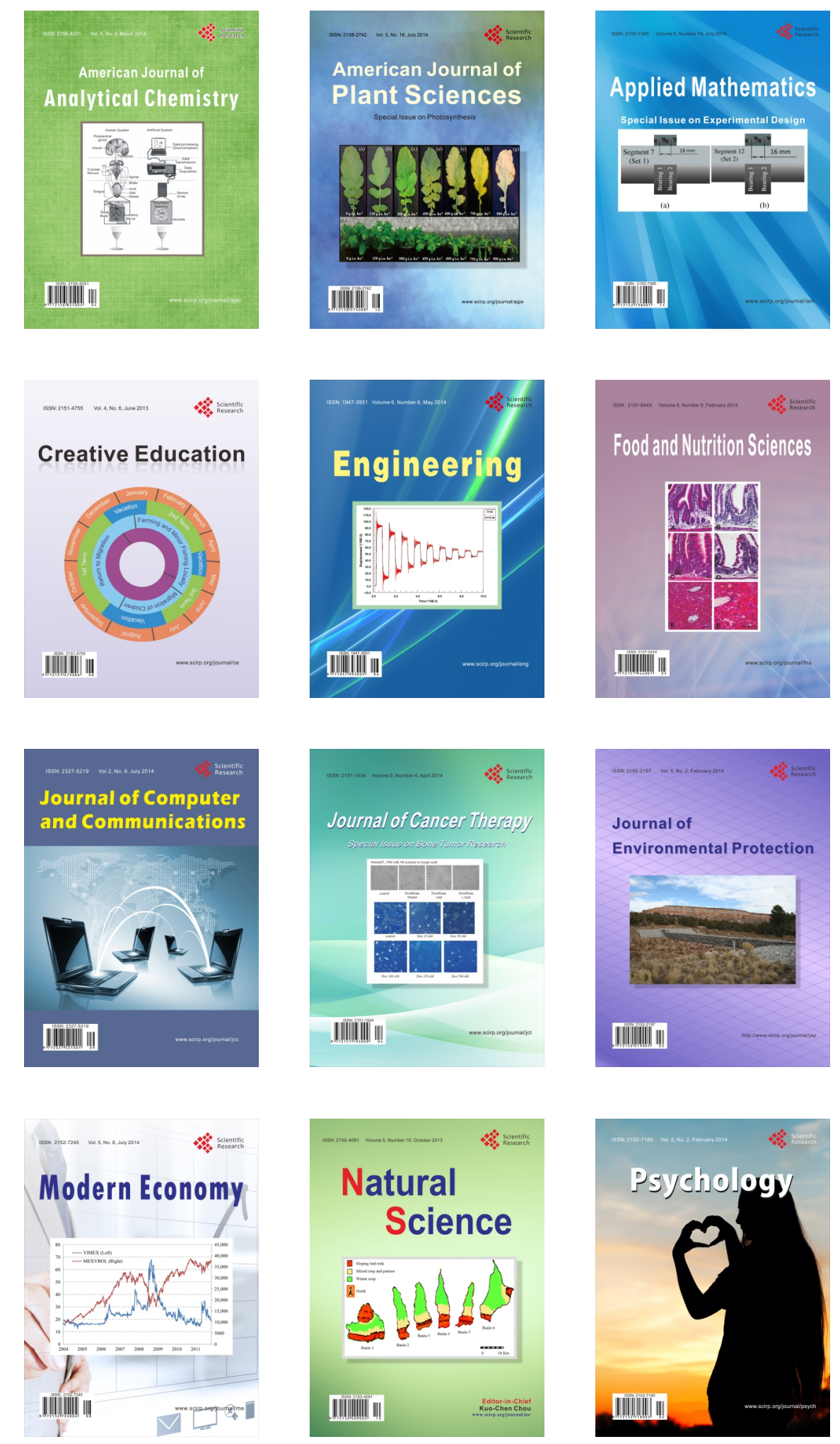